\title{
EXTENDED SPECTRUM B-LACTAMASE PRODUCTION AMONGST GRAM NEGATIVE BACILLI ISOLATED FROM PATIENTS ATTENDING A TERTIARY CARE HOSPITAL IN EASTERN BIHAR.
}

Krishan Nandan, Sangeeta Dey, Dhananjay Kumar, Aninda Sen, Udayan Ganguly

1. Assistant Professor, Department of Microbiology, Katihar Medical College, Katihar. Bihar.

2. Professor, Department of Microbiology, Katihar Medical College, Katihar. Bihar.

3. Assistant Professor, Department of Microbiology, Katihar Medical College, Katihar. Bihar.

4. Professor, Department of Microbiology, Katihar Medical College, Katihar. Bihar.

5. Professor, Department of Microbiology, Katihar Medical College, Katihar. Bihar.

\section{CORRESPONDING AUTHOR}

Dr. Krishan Nandan, Assistant Professor,

Department of Microbiology,

Katihar Medical College,

Karim Bagh, Katihar. Bihar 854105,

E-mail: drknandan@gmail.com,

Phone: +919431259633.

ABSTRACT: BACKGROUND: Extended Spectrum $\beta$-lactamases (ESBL) are enzymes that have the ability to hydrolyze $\beta$-lactam antibiotics containing oxyimino group and are inhibited by $\beta$ lactamase inhibitors. These enzymes are responsible for resistance to penicillins, monobactams, third generation and in some instances fourth generation cephalosporins. ESBLs are encoded by transferable conjugative plasmids that often code for resistance to other antibiotics as well. OBJECTIVE: This prospective study aimed to determine ESBL production in Gram negative bacteria in patients attending a tertiary care hospital in eastern Bihar. METHOD: A total of 556 samples from patients attending inpatient and outpatient departments from May 2009 to April 2010 were included in the study. Samples were processed as per standard protocol and antibiotic susceptibility testing was done by modified Kirby-Bauer method. Isolates showing resistance to any third generation cephalosporin were subjected to Double Disc Synergy Test (DDST), Phenotypic Confirmatory Disc Diffusion Test (PCDDT) and MIC reduction test for ESBL production. RESULTS: $42.62 \%$ of Gram negative bacilli were ESBL producers. $56.3 \%$ of Klebsiella pneumoniae strains were found to be ESBL producers whereas only $40.5 \%$ of Escherichia coli produced ESBL. PCDDT and MIC reduction test showed 100\% correlation whereas the DDST failed to detect $13.5 \%$ of ESBL producers. All ESBL producers were sensitive to Imipenem and Cefoperazone/ Sulbactam. CONCLUSION: The present study gives us an indication regarding the occurrence of ESBL producing Gram negative bacilli in Eastern Bihar. The number of ESBL producers in this region is alarmingly large. It is therefore recommended that PCDDT be incorporated in all laboratories as a part of routine antibiotic susceptibility testing procedures as it is simple, reliable and reproducible test for detection of ESBLs.

KEYWORDS: Extended Spectrum $\beta$-lactamases, ESBL, DDST, PCDDT.

INTRODUCTION: Extended Spectrum $\beta$-lactamases (ESBL) are enzymes that have the ability to hydrolyze $\beta$-lactam antibiotics containing an oxyimino group (third generation cephalosporin and Aztreonam) and are inhibited by $\beta$-lactamase inhibitors like Clavulanic acid, Sulbactam and tazobactam ${ }^{1}$. Production of ESBLs lead to resistance to penicillins, monobactams, third generation cephalosporins like Cefotaxime, Ceftriaxone, Ceftazidime and in some instances fourth generation cephalosporins also². 
Emergence of resistance to $\beta$-lactam antibiotics began even before the first $\beta$-lactam antibiotic penicillin was developed ${ }^{3}$. The first plasmid mediated $\beta$-lactamase, TEM- 1 was reported in 1965 from an Escherichia coli isolate belonging to a patient in Athens, Greece named Temoniera [hence designated TEM] ${ }^{4}$. Over the years, many new $\beta$-lactam antibiotics have been developed; however, with each new class of antibiotic, a new $\beta$-lactamase emerged that caused resistance to that class of drug. Presumably, the selective pressure imposed by the use and overuse of new antibiotics results in the emergence of new variants of $\beta$-lactamases ${ }^{5}$. The first report of plasmid-encoded $\beta$-lactamase capable of hydrolyzing the extended spectrum cephalosporins was published in 1983 from Germany6. Hence these new $\beta$-lactamases were coined as extended spectrum $\beta$-lactamases ${ }^{7}$. Over the past few decades, a number of new $\beta$ lactamases in clinical isolates of members of the family Enterobacteriaceae have emerged. The total number of ESBLs characterized exceeds 200 today8. ESBLs are encoded by transferable conjugative plasmids which often code for resistance to other antibiotics as well7. Being plasmid mediated, they are easily transmitted among the members of Enterobacteriaceae family, thus facilitating the dissemination of resistance not only to $\beta$ - lactams but also to other commonly used antibiotics such as quinolones and aminoglycosides 9 . Major risk factors for colonization or infection with ESBL producing organisms are prolonged exposure to antibiotics, prolonged ICU stay, nursing home residency, severe illness, catheterization, instrumental intervention and residence in an institution with high rate of use of third generation cephalosporins. ${ }^{5}$

This prospective study aimed at determining ESBL production in Gram negative bacilli in a tertiary care hospital in eastern Bihar. Detection of ESBL production is important because it ultimately determines the clinical outcome in patients infected with such strains. Studies on ESBL producing Gram negative bacilli have been reported from all parts of India however, to the best of our knowledge; no such studies till date have been reported from Bihar and Jharkhand.

MATERIAL AND METHODS: Clearance from Institutional Ethics Committee was obtained prior to carrying out this study. A total of five hundred and fifty six (556) samples viz. urine, pus, stool and pleural fluid from patients attending different inpatient and outpatient departments were included in the study. A brief clinical history of the patients regarding antibiotic intake, instrumentation and duration of hospital stay was taken. Specimens collected were inoculated on 5\% sheep blood agar and MacConkey's agar. They were identified by standard biochemical tests. ${ }^{10}$ Antibiotic Susceptibility Testing (AST) was done on Mueller-Hinton agar (MHA) plates by modified Kirby-Bauer disc diffusion technique using commercially available antibiotic discs (HiMedia, Mumbai). When the zone of inhibition of an isolate for any one or more of the third generation cephalosporin (Cefotaxime, Ceftriaxone, Ceftazidime \& Cefoperazone) was less than or equal to the zone diameter recommended by CLSI (Clinical and Laboratory Standards Institute), the isolate was further tested by the PCDDT, MIC reduction test and DDST for ESBL production. ${ }^{11}$

DDST: Bacterial suspension to be tested was prepared in Mueller-Hinton Broth (MHB). After matching the turbidity to 0.5 McFarland's standard, the organism was inoculated on MHA as per guidelines for disc diffusion technique. Amoxycillin/Clavulanic acid disc was placed in the centre of the plate. Four antibiotic discs i.e. Cefotaxime, Ceftazidime, Ceftriaxone and Cefoperazone were placed at a distance of $20 \mathrm{~mm}$ (centre to centre) from Amoxycillin/Clavulanic acid disc and at $90^{\circ}$ from each other. The plates were examined after 
overnight incubation. Enhancement of inhibition zone for any of the four antibiotic discs towards Amoxycillin/ Clavulanic acid disc indicated the production of ESBL by the strain.

PCDDT: MHA plates were inoculated in the same manner as DDST. Four antibiotic discs viz. Cefotaxime, Cefotaxime/ Clavulanic acid, Ceftazidime and Ceftazidime/ Clavulanic acid were placed at a distance of $30 \mathrm{~mm}$ (centre to centre) from each other. Plates were examined after overnight incubation at $37^{\circ} \mathrm{C}$. ESBL production was confirmed when there was an increase in the zone diameter by $5 \mathrm{~mm}$ or more when Clavulanic acid was added to the respective antibiotic. [Fig. 1]

MIC reduction test: Bacterial suspension to be tested was prepared in MHB and matched to 0.5 McFarland's standard. As a final concentration of $5 \times 10^{5} \mathrm{CFU} / \mathrm{ml}$ was required, bacterial suspension equivalent to 0.5 McFarland's standard was further diluted 1:100 in MHB. ${ }^{12}$

- Antibiotics in powder form were obtained from Sigma-Aldrich Chemicals Pvt. Ltd, Bangalore. Dilutions were prepared in the following range:

$\begin{array}{llc}\text { - } & \text { Ceftazidime } & 0.25-256 \mu \mathrm{g} / \mathrm{ml} \\ \text { - } & \text { Ceftazidime/Clavulanic acid } & : 0.25 / 4-256 / 4 \mu \mathrm{g} / \mathrm{ml} \\ \text { - } & \text { Cefotaxime } & 0.25-256 \mu \mathrm{g} / \mathrm{ml} \\ \text { - } & \text { Cefotaxime/Clavulanic acid }: & 0.25 / 4-256 / 4 \mu \mathrm{g} / \mathrm{ml}\end{array}$

Antibiotic ranges were prepared one step higher than the final dilution range required to compensate for the addition of equal volume of inoculum. $100 \mu \mathrm{l}$ of each antibiotic dilution was dispensed in their respective labelled wells. $100 \mu \mathrm{l}$ of diluted bacterial suspension was added to each well. $200 \mu \mathrm{l}$ of uninoculated and inoculated broth were also dispensed in wells in each row as sterility and growth control. After overnight incubation, the microtitre wells were examined. The lowest concentration of the antibiotic that showed no visible growth represented the MIC value of the organism. An isolate was confirmed as ESBL producer if there was $\geq 3$ two folds (eight times) reduction in MIC of third generation cephalosporin on addition of Clavulanic acid as compared to third generation cephalosporin when used alone ${ }^{12}$. [Fig. 2]

RESULTS: A total of five hundred and fifty six (556) samples were received in the laboratory during the study period out of which one hundred and twenty two strains of Gram negative bacilli were isolated (21.9\%). 62 strains were from indoor while 60 were from outdoor patients. Overall $52(42.6 \%)$ of these isolates were found to be ESBL producers; $56.3 \%$ of Klebsiella pneumoniae were ESBL producers, followed by $50.0 \%$ of Klebsiella oxytoca and $40.5 \%$ of Escherichia coli. ESBL production was also seen in Pseudomonas aeruginosa (35.7\%) and Proteus mirabilis (33.3\%). [Table 1]

Most of the ESBL strains were isolated from the department of Surgery (38.5\%) followed by department of Obstetrics \& Gynaecology (28.9\%) and department of Medicine (23.1\%). Comparison of DDST and PCDDT with MIC reduction test showed that the PCDDT was superior to the DDST for detection of ESBL producers. PCDDT showed $100 \%$ correlation with MIC reduction test whereas DDST failed to detect $13.5 \%$ of ESBL producers.

ESBL producers were isolated more frequently in patients hospitalized for more than 14 days (44.8\%) when compared with $\leq 7$ days of hospitalization (20.7\%). [Table 2]

All ESBL producers were resistant to Cefuroxime and Piperacillin. 90.0\% of strains were resistant to Cefoperazone, $82.7 \%$ to Ceftriaxone, $76.9 \%$ to cefotaxime and $75.0 \%$ to Ceftazidime. 
All the strains were sensitive to Imipenem and Cefoperazone/ Sulbactam combination. [Table 3]

DISCUSSION: The present study gives us an indication regarding the occurrence of ESBL producing Gram negative bacilli in Eastern Bihar. Unfortunately the number of ESBLs isolated in this region is alarmingly large ( $42.6 \%$ of all Gram negative bacilli). It was observed that majority of these ESBL producers were from indoor patients especially in those with prolonged hospital stay. Various studies have reported ESBL production to be as high as $61.7 \%$ and as low as $11.0 \% .^{13,14,15}$

Majority of ESBL producers were Klebsiella pneumoniae, this was followed by Klebsiella oxytoca and Escherichia coli. Many other studies have also reported that majority of ESBL producers were either Escherichia coli or Klebsiella pneumoniae.13 14,15 As was seen in this study, other authors have also reported ESBL production in Klebsiella oxytoca, Pseudomonas aeruginosa, Proteus mirabilis and Citrobacter freundii. $13,14,16,17$

The PCDDT was found to give good results as far as detection of ESBL was concerned, showing $100 \%$ concordance with the MIC reduction test. Similar findings were also reported by other workers. ${ }^{7}$

Presence of these organisms in the hospital environment is a man-made phenomenon due to over-use and misuse of $3^{\text {rd }}$ generation cephalosporins and other broad spectrum antibiotics. Proper infection control practices and formulation of a hospital antibiotic usage policy is clearly indicated. The other main issue that needs to be addressed is the incorporation of tests for detection of ESBL as a routine in all Microbiology laboratories. The PCDDT was found to be a simple, reliable and reproducible test that showed $100 \%$ conformity with the MIC reduction test which is time taking, difficult to interpret and needs a high degree of precision. The PCDDT therefore has the potential to be incorporated in all laboratories, especially smaller ones that do not have the resources to avail automated systems, as a part of routine antibiotic susceptibility testing procedures.

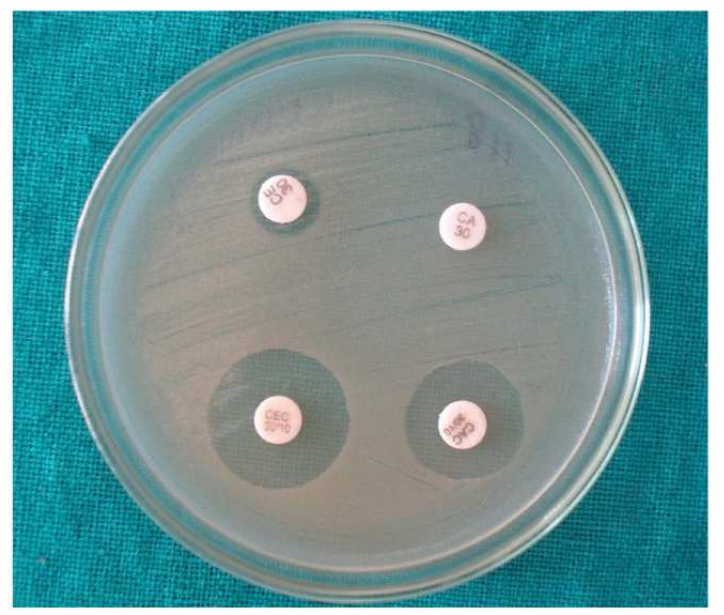

Fig.1 : Phenotypic Confirmatory Disc Diffusion Test showing enhancement of inhibition zone with corresponding disc containing clavulanic acid. 


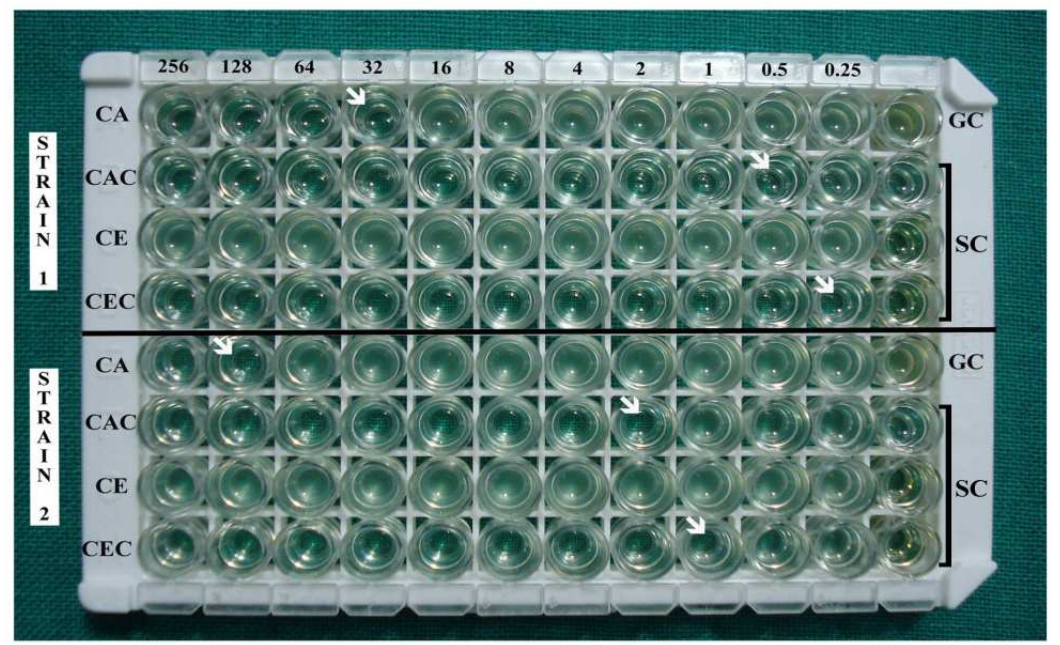

Fig.2 : Determination of Minimum Inhibitory Concentration by microdilution method.
CA: Ceftazidime CAC: Ceftazidime/clavulanic acid
CE: Cefotaxime
CEC: Cefotaxime/clavulanic acid
[arrows indicating MIC]
GC: Growth control
SC: Sterility control

Table 1: Rate of isolation of ESBL producers among Gram negative isolates.

\begin{tabular}{|l|c|c|c|}
\hline \multicolumn{1}{|c|}{ Isolate } & ESBL (\%) & Non-ESBL (\%) & Total \\
\hline Escherichia coli & $30(40.54)$ & $44(59.46)$ & 74 \\
\hline Klebsiella pneumonia & $09(56.25)$ & $07(43.75)$ & 16 \\
\hline Klebsiella oxytoca & $04(50.00)$ & $04(50.00)$ & 08 \\
\hline Pseudomonas aeruginosa & $05(35.71)$ & $09(64.29)$ & 14 \\
\hline Proteus mirabilis & $02(33.33)$ & $04(66.67)$ & 06 \\
\hline Citrobacter freundii & $02(50.00)$ & $02(50.00)$ & 04 \\
\hline Total & $52(42.62)$ & $70(57.38)$ & 122 \\
\hline
\end{tabular}


Table 2: Duration of hospital stay in relation with ESBL infection.

\begin{tabular}{|c|c|c|c|c|}
\hline Duration of stay (days) & ESBL & Percentage & Non-ESBL & Percentage \\
\hline$\leq 7$ & 06 & 20.69 & 22 & 66.67 \\
\hline $8-14$ & 10 & 34.48 & 06 & 18.18 \\
\hline$>14$ & 13 & 44.83 & 05 & 15.15 \\
\hline Total & 29 & 100.00 & 33 & 100.00 \\
\hline
\end{tabular}

Table 3: Antibiotic susceptibility pattern of ESBL and non-ESBL producing isolates.

\begin{tabular}{|l|l|c|c|c|c|}
\hline \multicolumn{1}{|c|}{ Antibiotics } & \multicolumn{1}{|c|}{$\begin{array}{c}\text { ESBL } \\
\text { production }\end{array}$} & $\begin{array}{c}\text { Resistant } \\
\mathbf{( \% )}\end{array}$ & $\begin{array}{c}\text { Intermediate } \\
\mathbf{( \% )}\end{array}$ & $\begin{array}{c}\text { Sensitive } \\
\mathbf{( \% )}\end{array}$ & Total \\
\hline Cefuroxime & ESBL & $52(100.0)$ & $0(0.00)$ & $0(0.0)$ & 52 \\
\cline { 2 - 6 } & Non ESBL & $58(82.9)$ & $11(15.71)$ & $01(1.4)$ & 70 \\
\hline Ceftazidime & ESBL & $39(75.0)$ & $13(25.00)$ & $0(0.0)$ & 52 \\
\cline { 2 - 6 } & Non ESBL & $18(25.7)$ & $10(14.29)$ & $42(60.0)$ & 70 \\
\hline Cefotaxime & ESBL & $40(76.9)$ & $10(19.23)$ & $02(3.8)$ & 52 \\
\cline { 2 - 6 } & Non ESBL & $22(31.4)$ & $09(12.86)$ & $39(55.7)$ & 70 \\
\hline Ceftriaxone & ESBL & $43(82.7)$ & $09(17.31)$ & $0(0.0)$ & 52 \\
\cline { 2 - 6 } & Non ESBL & $22(31.4)$ & $0(0.00)$ & $48(68.6)$ & 70 \\
\hline Cefoperazone & ESBL & $47(90.4)$ & $04(7.70)$ & $01(1.9)$ & 52 \\
\cline { 2 - 6 } & Non ESBL & $20(28.6)$ & $10(14.29)$ & $40(57.1)$ & 70 \\
\hline Levofloxacin & ESBL & $14(26.9)$ & $07(13.46)$ & $31(59.6)$ & 52 \\
\cline { 2 - 6 } & Non ESBL & $27(38.6)$ & $11(15.72)$ & $32(45.7)$ & 70 \\
\cline { 2 - 6 } & Non ESBL & $25(35.7)$ & $05(7.15)$ & $40(57.1)$ & 70 \\
\hline Amikacin & & & & & \\
\hline
\end{tabular}




\begin{tabular}{|l|l|c|c|c|c|}
\hline Gentamicin & ESBL & $36(69.2)$ & $01(1.92)$ & $15(28.9)$ & 52 \\
\cline { 2 - 6 } & Non ESBL & $41(58.6)$ & $09(12.86)$ & $20(28.6)$ & 70 \\
\hline Piperacillin & ESBL & $52(100.0)$ & $0(0.00)$ & $0(0.0)$ & 52 \\
\cline { 2 - 6 } & Non ESBL & $24(34.3)$ & $21(30.00)$ & $25(35.7)$ & 70 \\
\hline Cefoperazone/ & ESBL & $0(0.0)$ & $0(0.00)$ & $52(100.0)$ & 52 \\
\cline { 2 - 6 } Sulbactam & Non ESBL & $16(22.9)$ & $06(8.57)$ & $48(68.6)$ & 70 \\
\hline \multirow{2}{*}{ Tazobactam } & ESBL & $03(5.8)$ & $01(1.92)$ & $48(92.3)$ & 52 \\
\cline { 2 - 6 } & Non ESBL & $18(25.7)$ & $23(32.86)$ & $29(41.4)$ & 70 \\
\hline Imipenem & ESBL & $0(0.00)$ & $0(0.00)$ & $52(100.0)$ & 52 \\
\cline { 2 - 6 } & Non ESBL & $0(0.00)$ & $0(0.00)$ & $100(100.0)$ & 70 \\
\hline
\end{tabular}

\section{REFERENCES:}

1. Brooks GF and Carroll KC. Antimicrobial chemotherapy. In: Brooks GF, Carroll KC, Butel JS, Morse SA (eds). Jawetz, Melnick and Adelberg's Medical Microbiology, 24th ed. The McGraw-Hill Companies: New York: 2007; 162.

2. Russo AT and Johnson JR. Diseases caused by Gram negative enteric bacilli. In: Fauci AS, Kasper DL, Longo DL, Brawnwald E et al. (eds), Harrison's Principles of Internal Medicine, 17th ed, The McGraw Hill Companies: Boston: 2008; vol. I: 938.

3. Abraham EP and Chain E. An enzyme from bacteria able to destroy penicillin. Nature. 1940; $146: 837$.

4. Datta N and Kontomichalou P. Penicillinase synthesis controlled by infectious R Factors in Enterobacteriaceae. Nature. 1965; 208:239-244.

5. Chaudhary U and Aggarwal R. Extended spectrum beta lactamases (ESBL) - An emerging threat to clinical therapeutics. Indian J Med Microbiol. 2004; 22(2): 75-80.

6. Knothe H, Shah P, Krcmery V et al. Transferable resistance to cefotaxime, cefoxitin, cefamandole and cefuroxime in clinical isolates of Klebsiella pneumoniae and Serratia marcescens. Infection. 1983; 11: 315-317.

7. Agrawal Parul, Ghosh AN, Kumar Satish et al. Prevalence of extended spectrum $\beta$ lactamases among Escherichia coli and Klebsiella pneumoniae isolates in a tertiary care hospital. Indian J Pathol Microbiol. 2008; 51(1): 139-142.

8. Paterson David L and Bonomo Robert A. Extended spectrum $\beta$-lactamases: a clinical update. Clin Microbiol Rev. 2005; 18(4): 657-686.

9. Varaiya A, Dogra J. Kulkarni $M$ et al. Extended spectrum $\beta$-lactamase producing Escherichia coli and Klebsiella pneumoniae in diabetic foot infection. Indian J Med Microbiol. 2008; 26(3): 281-282. 
10. Forbes BA, Sahm DF, Weissfeld AS (eds). Overview of Bacterial Identification Methods and Strategies. In: Bailey and Scott's Diagnostic Microbiology. $12^{\text {th }}$ ed. Mosby Elsevier, Missouri. 2007; 216-247.

11. CLSI. Performance standards for antimicrobial susceptibility testing: Eighteenth informational supplement, M100-S18, January. 2008; 28(1): 162-163.

12. Andrews JM. Determination of minimum inhibitory concentration. J Antimicrobiol Chemother.2001; 44: 5-16.

13. Deep and Usha Arora. Extended spectrum $\beta$-lactamases - mediated resistance among the family Enterobacteriaceae. Indian J Pathol Microbiol. 2007; 50(2): 458-461.

14. Sridhar Rao PN, Basavarajappa KG and Krishna G. Leela. Detection of extended spectrum beta-lactamase from clinical isolates in Davangere. Indian J Pathol Microbiol. 2008; 51(4): 497-499.

15. Kader Abdulrahman Abdulla and Kumar Angamuthu. Prevalence and antimicrobial susceptibility of extended spectrum beta-lactamase-producing Escherichia coli and Klebsiella pneumoniae in a general hospital. Ann Saudi Med. 2005; 25(3): 239-242.

16. Aggrawal Ritu, Chaudhary Uma and Sikka Rama. Detection of extended spectrum $\beta$ lactamase production among uropathogens. J Lab Physicians. 2009; 1(1): 7-9.

17. Bhattacharjee A, MR Sen, P Prakash et al. Increased Prevalence of Extended Spectrum Beta lactamase producers in neonatal septicemia cases at a tertiary referral hospital. Indian J Med Microbiol. 2008; 26(4): 356-360. 